\title{
Rig Animation with a Tangible and Modular Input Device
}

\author{
Oliver Glauser \\ ETH Zurich \\ oliver.glauser@inf.ethz.ch
}

\author{
Daniele Panozzo \\ New York University
}

\author{
Benedek Vartok \\ ETH Zurich
}

\author{
Alec Jacobson \\ Columbia University
Olga Sorkine-Hornung
ETH Zurich

\author{
Wan-Chun Ma \\ Activision, Inc.
}

\author{
Otmar Hilliges \\ ETH Zurich
}

\begin{abstract}
We propose a novel approach to digital character animation, combining the benefits of modular and tangible input devices and sophisticated rig animation algorithms. With a symbiotic software and hardware approach, we overcome limitations inherent to all previous tangible devices. It allows users to directly control complex rigs with 5-10 physical controls only. These compact input device configurations - optimized for a specific rig and a set of sample poses - are automatically generated by our algorithm. This avoids oversimplification of the pose space and excessively bulky devices.
\end{abstract}

\section{Author Keywords}

Novel input devices; character articulation

\section{ACM Classification Keywords}

H.5.2 Information Interfaces \& Presentation: User Interfaces

\section{INTRODUCTION}

For interactive character animation - as an alternative to classic mouse/keyboard interfaces - recent tangible input devices promise direct and natural manipulation, but at the cost of either grossly simplifying the pose space or of accepting complex and bulky physical setups. In contrast, we present a novel software/hardware approach co-designed to help animators traverse a large space of poses via fluid manipulation of a tangible controller.

Specifically, we contribute: (a) an algorithm to compute a device configuration and instructions to assemble it, using only a small set of modules; (b) a novel hardware design, overcoming major limitations in prior work via a novel physical angle parametrization; and (c) a method to bridge the disparity between the input device's few degrees of freedom to the character's many control parameters.

Permission to make digital or hard copies of part or all of this work for personal or classroom use is granted without fee provided that copies are not made or distributed for profit or commercial advantage and that copies bear this notice and the full citation on the first page. Copyrights for third-party components of this work must be honored. For all other uses, contact the Owner/Author

Copyright is held by the owner/author(s)

UIST'16 Adjunct, October 16-19, 2016, Tokyo, Japan

ACM 978-1-4503-4531-6/16/10.

http://dx.doi.org/10.1145/2984751.2985696

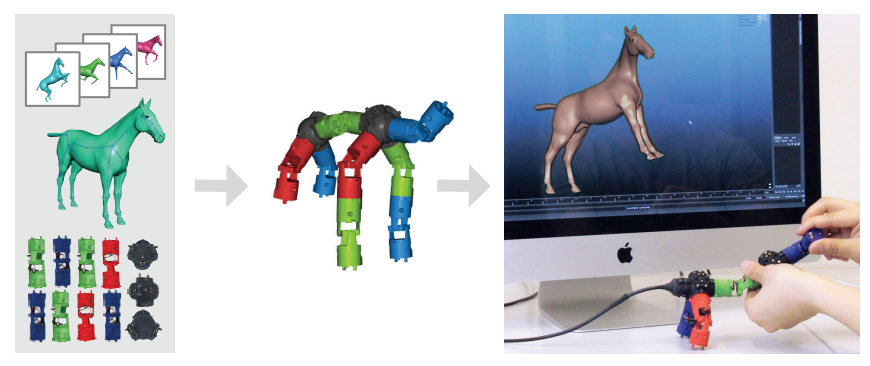

Figure 1. Illustration of our pipeline from input character to fluid tangible animation. The horse has 29 bones, controlled by 8 joints.

Our approach (as described in detail in Glauser et al. [1]) allows novice users and experts to create animation sequences. Results from a user study (see [1]) compare favorably to the hardware design of Jacobson et al. [2] both in terms of accuracy and posing time, providing an average speed-up of $2 \times$. The method is integrated directly into Autodesk's Maya ${ }^{\circledR} 3 \mathrm{D}$ animation software and its open-source counterpart Blender.

\section{TECHNICAL CONTRIBUTION}

The user provides a rigged 3D character with a sparse set of sample poses (readily available online). Furthermore, the user indicates the kit (number of joints and splitters) to use (Figure 1, left). Our algorithm then analyzes the rig and the poses, identifying the DoFs with the most influence on pose reachability, weighted by the amount of controlled surface. Optimizing for direct control of these most important nodes, and using only the available parts, a device configuration and assembly instructions are computed (Figure 1, middle). After assembly the compact physical device is automatically bound to the virtual rig and user inputs are mapped onto the rig. Manipulating the device induces a similar deformation onto the 3D character (Figure 1, right). In most cases the physical configuration has significantly fewer DoFs than the rig while still allowing to cover a large enough pose space as shown in Figure 2. To maintain expressiveness and add details back into the resulting motion, we use a pose space interpolation scheme to synthesize detailed pose nuances.

Our hardware design, inspired by Jacobson et al. [2], follows a modular approach, decomposing the control structure into 


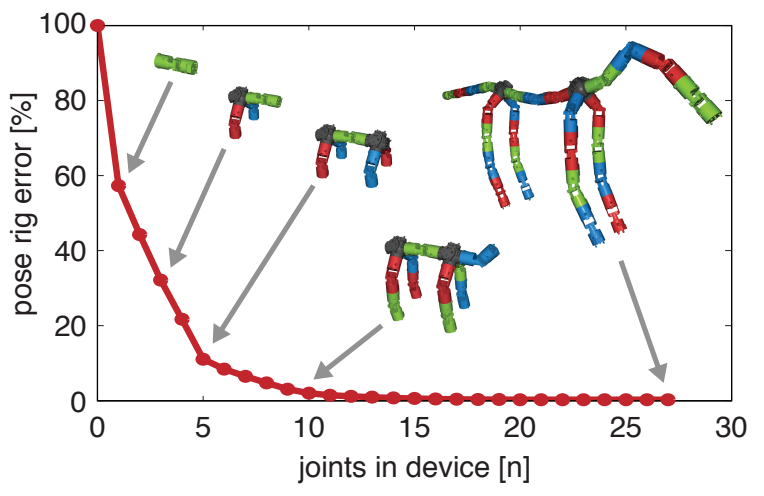

Figure 2. Influence of joint number in the kit on pose accuracy. Generated devices for different kits are shown: A device of 12 parts $(10$ joints and 2 splitters) allows to reduce the sample pose error by $98 \%$.

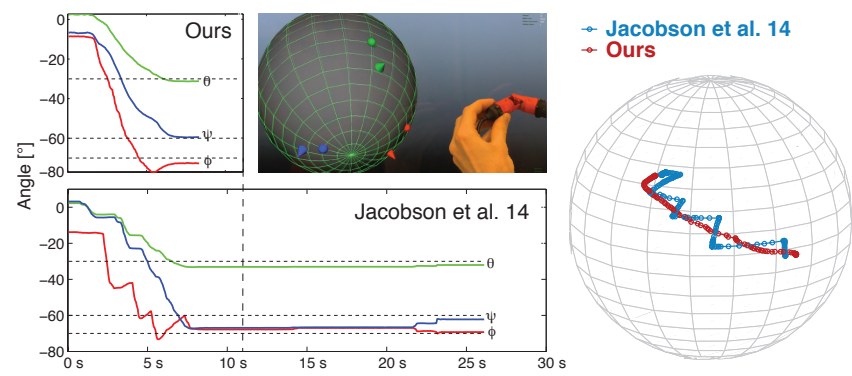

Figure 3. Our physical angle parametrization allows for direct tracing of geodesics. The joints of Jacobson et al. [2] force angular decomposition of rotations, resulting in a zig-zag pattern. Ours is also significantly faster in reaching the target position.

joints that measure 3D rotations, and splitters, which allow for branching. This design enables dynamic rearrangement of the parts into arbitrary topologies. However, one of the main limitations of [2] is due to the mechanical joint design. We propose a new joint design to overcome this limitation and provide a much improved user-experience by allowing for smoother and faster tracing of geodesics - both for individual joints (see Figure 3) and chains of joints. More details on our novel joint design can be found in [1].

\section{ENHANCEMENTS}

Since Glauser et al. [1] we extended the software periphery of the input device with a Blender plugin that offers a much improved user interaction, e.g., an interactive assembly guide. And using an RBF (radial basis function) interpolation scheme (inspired by Sloan et al. [3]) leads to smoother detailed pose nuances.

\section{DEMO}

Visitors have the opportunity to try our novel tangible and modular user input device (example sequence in Figure 5). With this tangible interface even visitors without any prior experience in 3D animation are able to create short character animation sequences in Blender.

Thanks to the modularity of the device, the visitor can choose from a set of 3D characters. Additionally the size of the kit (joints and splitters) can be varied. Based on these inputs our

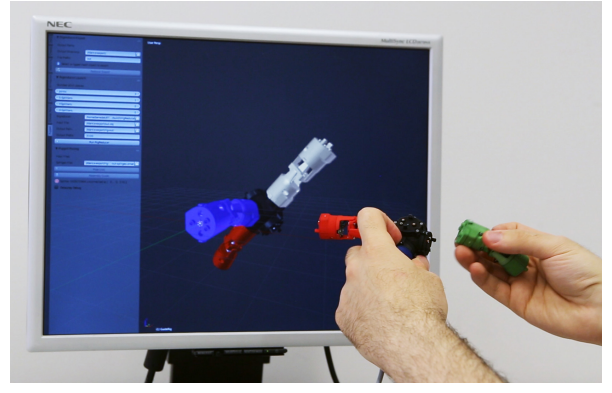

Figure 4. Interactive assembly of the optimized device in Blender: The user is interactively guided in assembling the optimized device by having the already correctly connected parts of the device displayed in color and the ones still missing in light grey.

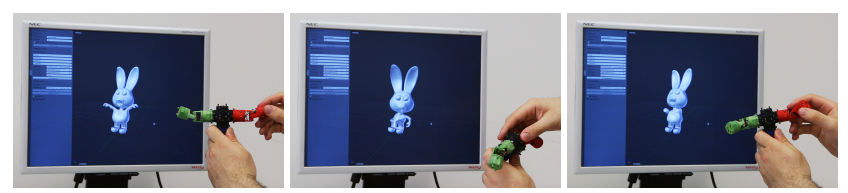

Figure 5. Example posing session of a bunny character, with a device consisting of 3 joints and 1 splitter.

novel algorithm suggests an optimized device configuration and interactive assembly instructions (see Figure 4) for the visitor.

We demonstrate how this optimized compact device with few degrees of control is sufficient to pose a complex rig with many degrees of freedom. It also allows to experience how the novel joint design enables a more fluid and direct control compared to state-of-the-art devices.

For easy reproducibility and to foster future work, we are releasing the open-hardware specification and the software implementations ${ }^{1}$. For footage of the input device in action and more results, we refer to the accompanying video.

\section{ACKNOWLEDGMENTS}

This work was supported in part by the SNF grant 200021_162958 and the ERC grant iModel (StG-2012306877). Alec Jacobson is funded in part by NSF grants IIS14-09286 and IIS-17257.

\section{REFERENCES}

1. Glauser, O., Ma, W.-C., Panozzo, D., Jacobson, A., Hilliges, O., and Sorkine-Hornung, O. Rig Animation with a Tangible and Modular Input Device. ACM Trans. Graph. 35, 4 (2016).

2. Jacobson, A., Panozzo, D., Glauser, O., Pradalier, C., Hilliges, O., and Sorkine-Hornung, O. Tangible and modular input device for character articulation. $A C M$ Trans. Graph. 33, 4 (2014).

3. Sloan, P.-P. J., Rose, III, C. F., and Cohen, M. F. Shape by example. In Proceedings of the 2001 Symposium on Interactive 3D Graphics, I3D '01, ACM (New York, NY, USA, 2001), 135-143.

\footnotetext{
${ }^{1}$ http://oliglauser.github.io/atamid/
} 